\title{
On Filter-regular Sequences of Multi-graded Modules
}

\author{
Duong Quoc VIET and Truong Thi Hong THANH \\ Hanoi National University of Education \\ (Communicated by K. Ahara)
}

\begin{abstract}
Let $S$ be an $\mathbf{N}^{d}$-graded algebra over a noetherian ring and a finitely generated $\mathbf{N}^{d}$-graded $S$-module $M$. This paper will study the relationship of filter-regular sequences of $M$ to joint reductions and homogeneous parameter systems. As an application, we show that any maximal filter-regular sequence is a joint reduction of $\left(S_{1}, \ldots, S_{d}\right)$ with respect to $M$, and any maximal strong-filter-regular sequence is a reduction of $S_{+}$with respect to $M$. And we characterize the existence of parts of homogeneous parameter systems for $M$ consisting of elements of total degree 1 via strong-filter-regular sequences.
\end{abstract}

\section{Introduction}

Throughout this paper, let $(A, \mathfrak{m})$ be a noetherian local ring with maximal ideal $\mathfrak{m}$, infinite residue field $k=A / \mathfrak{m}$. Let $S=\bigoplus_{n_{1}, \ldots, n_{d} \geq 0} S_{\left(n_{1}, \ldots, n_{d}\right)}$ be a finitely generated standard $\mathbf{N}^{d}$-graded algebra over $A$, i.e., $S$ is generated over $A$ by elements of total degree 1. Let $M=\bigoplus_{n_{1}, \ldots, n_{d} \geq 0} M_{\left(n_{1}, \ldots, n_{d}\right)}$ be a finitely generated $\mathbf{N}^{d}$-graded $S$-module that satisfies $M_{\left(n_{1}, \ldots, n_{d}\right)}=S_{\left(n_{1}, \ldots, n_{d}\right)} M_{(0, \ldots, 0)}$ for all $n_{1}, \ldots, n_{d} \geq 0$. Set

$$
S_{+}=\bigoplus_{n_{1}+\cdots+n_{d}>0} S_{\left(n_{1}, \ldots, n_{d}\right)} ; S_{++}=\bigoplus_{n_{1}, \ldots, n_{d}>0} S_{\left(n_{1}, \ldots, n_{d}\right)} ; S_{i}=S_{(\underbrace{0, \ldots, 1}_{i}, \ldots, 0)}
$$

for all $i=1, \ldots, d$.

In this paper, we explore the relation of filter-regular sequences in multi-graded modules with superficial sequences of ideals in local rings; joint reductions; and parts of homogeneous parameter systems for $M$.

Filter-regular sequences were introduced by Stuckrad and Vogel in [12]. The theory of these sequences became as an important tool to study some classes of singular rings and has been continually developed (see e.g. [1, 5, 14, 22, 25]).

DEFINITION 1.1 (Definition 2.1). Let $M$ be a finitely generated $\mathbf{N}^{d}$-graded $S$-module.

Received May 15, 2014; revised August 30, 2014

2010 Mathematics Subject Classification: 13A02 (Primary), 16W50, 13A15, 13 E05 (Secondary)

Key words and phrases: Filter-regular sequence, multi-graded module, joint reduction, parameter system

This research was in part supported by a grant from NAFOSTED. 
(i) Let $S_{(1,1, \ldots, 1)}$ be not contained in $\sqrt{\mathrm{Ann} M}$. A homogeneous element $x \in S$ is called an $S_{++}$-filter-regular element with respect to $M$ if $\left(0_{M}: x\right)_{\left(n_{1}, \ldots, n_{d}\right)}=0$ for all large $n_{1}, \ldots, n_{d}$.

(ii) Let $S_{i}$ be not contained in $\sqrt{\operatorname{Ann} M}$. A homogeneous element $x \in S$ is called an $S_{i}$-filter-regular element with respect to $M$ if for $n_{1}, \ldots, n_{i-1}, n_{i+1}, \ldots, n_{d} \geq 0$ there exists $N \in \mathbf{N}$ such that $\left(0_{M}: x\right)_{\left(n_{1}, \ldots, n_{i}, \ldots, n_{d}\right)}=0$ for all $n_{i} \geq N$.

We first study the relation of filter-regular sequences with joint reductions.

The concept of joint reductions of m-primary ideals was given by Rees in 1984 [10]. This important concept was extended to the set of arbitrary ideals by [2, 7, 9, 17, 19, 21]. In the case of graded modules, we have the following notion.

Definition 1.2 (Definition 3.1). Let $\mathfrak{R}$ be a subset of $\bigcup_{i=1}^{d} S_{i}$. Set $\mathfrak{R}_{i}=\mathfrak{R} \bigcap S_{i}$ for $1 \leqslant i \leqslant d$. Then $\Re$ is called a joint reduction of $\left(S_{1}, \ldots, S_{d}\right)$ with respect to $M$ if for all large integers $n_{1}, n_{2}, \ldots, n_{d}, M_{\left(n_{1}+1, \ldots, n_{d}+1\right)}=\sum_{i=1}^{d}\left(\Re_{i}\right) M_{\left(n_{1}+1, \ldots, n_{i}, \ldots, n_{d}+1\right)}$.

Then we obtain the following interesting result.

THEOREM 1.3 (Theorem 3.2). Let $\mathfrak{R}$ be a subset of $\bigcup_{i=1}^{d} S_{i}$. Then the following statements hold.

(i) If $\Re$ is a maximal $S_{++}$-filter-regular sequence with respect to $M$, then $\Re$ is a joint reduction of $\left(S_{1}, \ldots, S_{d}\right)$ with respect to $M$.

(ii) If $\mathfrak{R}$ is a maximal strong-filter-regular sequence with respect to $M$, then $(\mathfrak{R})$ is a reduction of $S_{+}$with respect to $M$.

As applications of Theorem 1.3 for joint reductions of ideals, we show that any maximal weak-(FC)-sequence is a joint reduction (Corollary 3.5, Section 3); and in the case of $\mathfrak{m}$ primary ideals, each maximal superficial sequence is also a joint reduction (Corollary 3.6, Section 3).

It is well known that one of obstructions in studying graded modules is that these modules often do not have homogeneous parameter systems. Using strong-filter-regular sequences, we characterize the existence of parts of homogeneous parameter systems for $M$ consisting of elements of total degree 1 by the following theorem.

THEOREM 1.4 (Theorem 4.1). Set $\mathfrak{S}_{\hat{i}}=\left\{S_{1}, \ldots, S_{i-1}, S_{i+1}, \ldots, S_{d}\right\}$ for $1 \leq i \leq$ $d$. Let $k_{1}, k_{2}, \ldots, k_{d}$ be non-negative integers with $k_{1}+k_{2}+\cdots+k_{d}=s \leq \operatorname{dim} M$. Then the following statements are equivalent.

(i) For any $1 \leq i \leq d, \operatorname{dim}_{S} \frac{M}{\left(\mathfrak{U}, S_{i}\right) M} \leq \operatorname{dim}_{S} \frac{M}{(\mathfrak{U}) M}-k_{i}$ for all $\mathfrak{U} \subset \mathfrak{S}_{\hat{i}}$.

(ii) There is a strong-filter-regular sequence $x_{1}, \ldots, x_{s}$ with respect to $M$ consisting of $k_{1}$ elements of $S_{1}, \ldots, k_{d}$ elements of $S_{d}$ such that if $y_{1}, \ldots, y_{k_{i}} \in S_{i}$ is a subsequence of $x_{1}, \ldots, x_{s}$, then $\operatorname{dim}_{S} \frac{M}{\left(\mathfrak{U}, y_{1}, \ldots, y_{k_{i}}\right) M}=\operatorname{dim}_{S} \frac{M}{(\mathfrak{U}) M}-k_{i}$ for all $\mathfrak{U} \subset \mathfrak{S}_{\vec{i}}$ and $1 \leq i \leq d$. And $x_{1}, \ldots, x_{s}$ is a part of a parameter system for $M$. 
(iii) There is a part of a parameter system $x_{1}, \ldots, x_{s}$ for $M$ consisting of $k_{1}$ elements of $S_{1}, \ldots, k_{d}$ elements of $S_{d}$ such that if $y_{1}, \ldots, y_{k_{i}} \in S_{i}$ is a subsequence of $x_{1}, \ldots, x_{s}$, then $\operatorname{dim}_{S} \frac{M}{\left(\mathfrak{U}, y_{1}, \ldots, y_{k_{i}}\right) M}=\operatorname{dim}_{S} \frac{M}{(\mathfrak{U}) M}-k_{i}$ for all $\mathfrak{U} \subset \mathfrak{S}_{\hat{i}}$ and $1 \leq i \leq$ $d$.

Theorem 1.4 yields interesting consequences such as the existence of homogeneous parameter ideals generated by elements of total degree 1 for $\mathbf{N}^{d}$-graded modules over artinian rings (Corollary 4.7; Corollary 4.8, Section 4) and different consequences and examples (Corollary 4.2; Corollary 4.5; Example 4.4; Example 4.6).

This paper is divided into four sections. Section 2 is devoted to the discussion of filterregular sequences and strong-filter-regular sequences such as the universal existence of strongfilter-regular sequences; the relationship of filter-regular sequences of multi-graded modules with superficial sequences and weak-(FC)-sequences of ideals; and other properties that will be used in this paper. Section 3 explores the relationship between maximal filter-regular sequences and joint reductions and gives some applications for joint reductions of ideals. In Section 4 we characterize the existence of parts of homogeneous parameter systems for $M$. As an application we get some results in the case of artinian rings.

\section{Filter-Regular Sequences}

In this section, we will define the notion of filter-regular sequences and strong-filterregular sequences in multi-graded modules, and study some important properties of these sequences.

Definition 2.1. Let $S=\bigoplus_{n_{1}, \ldots, n_{d} \geq 0} S_{\left(n_{1}, \ldots, n_{d}\right)}$ be a finitely generated standard $\mathbf{N}^{d}$ graded algebra over a noetherian local ring $A$ and let $M=\bigoplus_{n_{1}, \ldots, n_{d} \geq 0} M_{\left(n_{1}, \ldots, n_{d}\right)}$ be a finitely generated $\mathbf{N}^{d}$-graded $S$-module. Then we have the following notions:

(i) Let $S_{(1,1, \ldots, 1)}$ be not contained in $\sqrt{\mathrm{Ann} M}$. A homogeneous element $x \in S$ is called an $S_{++}$-filter-regular element with respect to $M$ if $\left(0_{M}: x\right)_{\left(n_{1}, \ldots, n_{d}\right)}=0$ for all large $n_{1}, \ldots, n_{d}$. Let $x_{1}, \ldots, x_{t}$ be homogeneous elements in $S$. We call that $x_{1}, \ldots, x_{t}$ is an $S_{++}$-filter-regular sequence with respect to $M$ if $x_{i+1}$ is an $S_{++}{ }^{-}$ filter-regular element with respect to $M_{i}=\frac{M}{\left(x_{1}, \ldots, x_{i}\right) M}$ for all $i=0, \ldots, t-1$.

An $S_{++}$-filter-regular sequence $x_{1}, \ldots, x_{t}$ with respect to $M$ is called a maximal $S_{++}$-filter-regular sequence if $S_{(1,1, \ldots, 1)} \subseteq \sqrt{\mathrm{Ann} M_{t}}$.

(ii) Let $S_{i}$ be not contained in $\sqrt{\operatorname{Ann} M}$. A homogeneous element $x \in S$ is called an $S_{i}$ filter-regular element with respect to $M$ if for $n_{1}, \ldots, n_{i-1}, n_{i+1}, \ldots, n_{d} \geq 0$ there exists $N \in \mathbf{N}$ such that $\left(0_{M}: x\right)_{\left(n_{1}, \ldots, n_{i}, \ldots, n_{d}\right)}=0$ for all $n_{i} \geq N$. Let $x_{1}, \ldots, x_{t}$ be homogeneous elements in $S$. We call that $x_{1}, \ldots, x_{t}$ is a strong-filter-regular sequence with respect to $M$ if $x_{i+1}$ is an $S_{j}$-filter-regular element for some $j$ with respect to $M_{i}=\frac{M}{\left(x_{1}, \ldots, x_{i}\right) M}$ for all $i=0, \ldots, t-1$. 
A strong-filter-regular sequence $x_{1}, \ldots, x_{t}$ with respect to $M$ is called a maximal strong-filter-regular sequence if $S_{i} \subseteq \sqrt{\mathrm{Ann} M_{t}}$ for all $i=1, \ldots, d$.

REMARK 2.2. We have some following comments for filter-regular sequences.

(i) $S_{(1,1, \ldots, 1)} \subseteq \sqrt{\mathrm{Ann} M}$ if and only if there exists $u>0$ such that $S_{(u, \ldots, u)} M=0$. Since $S_{(u, \ldots, u)} M_{\left(m_{1}, \ldots, m_{d}\right)}=M_{\left(m_{1}+u, \ldots, m_{d}+u\right)}$, it follows that $S_{(1,1, \ldots, 1)} \subseteq \sqrt{\operatorname{Ann} M}$ if and only if $M_{\left(n_{1}, \ldots, n_{d}\right)}=0$ for all $n_{1}, \ldots, n_{d} \gg 0$. Then in this case, we get $\left(0_{M}: x\right)_{\left(n_{1}, \ldots, n_{d}\right)} \subseteq M_{\left(n_{1}, \ldots, n_{d}\right)}=0$ for all large $n_{1}, \ldots, n_{d}$. Hence any homogeneous element of $S$ has the property as an $S_{++}$-filter-regular element.

If $S_{i} \subseteq \sqrt{\operatorname{Ann} M}$ then $S_{\left(0, \ldots, a_{i}, \ldots, 0\right)} M=0$ for some $a_{i}$. Hence

$$
M_{\left(n_{1}, \ldots, n_{i}+a_{i}, \ldots, n_{d}\right)}=S_{\left(0, \ldots, a_{i}, \ldots, 0\right)} M_{\left(n_{1}, \ldots, n_{d}\right)}=0
$$

for all $n_{1}, \ldots, n_{d}$. Thus $\left(0_{M}: x\right)_{\left(n_{1}, \ldots, n_{d}\right)} \subseteq M_{\left(n_{1}, \ldots, n_{d}\right)}=0$ for all $n_{i} \gg 0$ and for all $n_{1}, \ldots, n_{i-1}, n_{i+1}, \ldots, n_{d} \geq 0$. In this case, any homogeneous element of $S$ carries the property of an $S_{i}$-filter-regular element.

The above facts only obstruct and do not carry usefulness. That is why in Definition 2.1, one has to exclude the cases that $S_{(1,1, \ldots, 1)} \subseteq \sqrt{\operatorname{Ann} M}$ and $S_{i} \subseteq \sqrt{\mathrm{Ann} M}$ in defining $S_{++}$-filter-regular elements and $S_{i}$-filter-regular elements, respectively.

(ii) By [22], a homogeneous element $x \in S$ is an $S_{++}$-filter-regular element with respect to $M$ if and only if $0_{M}: x \subseteq 0_{M}: S_{++}^{\infty}$.

(iii) One showed that if $S_{(1,1, \ldots, 1)}$ is not contained in $\sqrt{\mathrm{Ann} M}$ then for each $i=$ $1, \ldots, d$, there exists an $S_{++}$-filter-regular element $x \in S_{i}$ with respect to $M$ (see [22, Proposition 2.2]).

Next, we show that the existence of strong-filter-regular sequences is universal by the following proposition.

Proposition 2.3. Assume that $S_{i}$ is not contained in $\sqrt{\mathrm{AnnM}}$. Then there exists an $S_{i}$-filter-regular element $y \in S_{i}$ with respect to $M$. And a homogeneous element $x$ is an $S_{i}$ filter-regular element with respect to $M$ if and only if $0_{M}: x \subseteq 0_{M}: S_{i}^{\infty}$.

Proof. Note that since $M$ is noetherian, there exists a positive integer $n$ such that $0_{M}: S_{i}^{\infty}=0_{M}: S_{i}^{n}$. For any $a_{1}, \ldots, a_{i-1}, a_{i+1}, \ldots, a_{d} \geq 0$, set

$$
M^{\prime}=\left[0_{M}: S_{i}^{n}\right] \bigcap\left[\bigoplus_{n_{i} \geq 0} M_{\left(a_{1}, \ldots, a_{i-1}, n_{i}, a_{i+1}, \ldots, a_{d}\right)}\right] .
$$

Then $M^{\prime}$ is a noetherian graded $\left[\bigoplus_{n_{i} \geq 0} S_{\left(0, \ldots, n_{i}, \ldots, 0\right)}\right]$-submodule of

$$
\bigoplus_{n_{i} \geq 0} M_{\left(a_{1}, \ldots, a_{i-1}, n_{i}, a_{i+1}, \ldots, a_{d}\right)} .
$$


Hence by [22, Lemma 2.3], there exists a positive integer $u_{i}$ such that

$$
M_{\left(a_{1}, \ldots, a_{i-1}, n_{i}, a_{i+1}, \ldots, a_{d}\right)}^{\prime}=S_{\left(0, \ldots, n_{i}-u_{i}, \ldots, 0\right)} M_{\left(a_{1}, \ldots, a_{i-1}, u_{i}, a_{i+1}, \ldots, a_{d}\right)}^{\prime}
$$

for all $n_{i} \geq u_{i}$. Consequently

$$
\left[0_{M}: S_{i}^{n}\right]_{\left(a_{1}, \ldots, a_{i-1}, n_{i}, a_{i+1}, \ldots, a_{d}\right)}=S_{\left(0, \ldots, n_{i}-u_{i}, \ldots, 0\right)}\left[0_{M}: S_{i}^{n}\right]_{\left(a_{1}, \ldots, a_{i-1}, u_{i}, a_{i+1}, \ldots, a_{d}\right)}
$$

for all $n_{i} \geq u_{i}$. From this fact follows that

$$
\left[0_{M}: S_{i}^{n}\right]_{\left(a_{1}, \ldots, a_{i-1}, n_{i}, a_{i+1}, \ldots, a_{d}\right)}=0
$$

for all $n_{i} \geq n+u_{i}$. So for any $a_{1}, \ldots, a_{i-1}, a_{i+1}, \ldots, a_{d} \geq 0$ we have

$$
\left[0_{M}: S_{i}^{\infty}\right]_{\left(a_{1}, \ldots, a_{i-1}, n_{i}, a_{i+1}, \ldots, a_{d}\right)}=0
$$

for all $n_{i} \gg 0$. Now note that

$$
\operatorname{Ass}_{S}\left(M / 0_{M}: S_{i}^{\infty}\right)=\left\{P \in \operatorname{Ass}_{S} M \mid S_{i} \nsubseteq P\right\} .
$$

Since $k$ is an infinite field and $\amalg=\operatorname{Ass}_{S}\left(M / 0_{M}: S_{i}^{\infty}\right)$ is finite, there exists $y \in S_{i} \backslash \bigcup_{P \in \amalg} P$. Then $0_{M}: y \subseteq 0_{M}: S_{i}^{\infty}$. Therefore, for $a_{1}, \ldots, a_{i-1}, a_{i+1}, \ldots, a_{d} \geq 0$ we obtain

$$
\left[0_{M}: y\right]_{\left(a_{1}, \ldots, a_{i-1}, n_{i}, a_{i+1}, \ldots, a_{d}\right)} \subseteq\left[0_{M}: S_{i}^{\infty}\right]_{\left(a_{1}, \ldots, a_{i-1}, n_{i}, a_{i+1}, \ldots, a_{d}\right)}=0
$$

for all $n_{i} \gg 0$. Thus $y$ is an $S_{i}$-filter-regular element with respect to $M$.

Next, it has already been shown that if a homogeneous element $x$ satisfies

$$
0_{M}: x \subseteq 0_{M}: S_{i}^{\infty}
$$

then $x$ is an $S_{i}$-filter-regular element with respect to $M$. Now assume that $x$ is an $S_{i}$-filterregular element with respect to $M$. We need to show that

$$
0_{M}: x \subseteq 0_{M}: S_{i}^{\infty} .
$$

It is enough to prove that $\left(0_{M}: x\right)_{\left(v_{1}, \ldots, v_{d}\right)} \subseteq 0_{M}: S_{i}^{\infty}$ for all $v_{1}, \ldots, v_{d}$. Indeed, for any $z \in\left(0_{M}: x\right)_{\left(v_{1}, \ldots, v_{d}\right)}$ then $x S_{\left(n_{1}, \ldots, n_{d}\right)} z=0$ for every $n_{1}, \ldots, n_{d}$. Hence

$$
S_{i}^{n} z \subseteq\left(0_{M}: x\right)_{\left(v_{1}, \ldots, v_{i-1}, n+v_{i}, v_{i+1}, \ldots, v_{d}\right)}=0
$$

for all large $n$. It implies that $z \in 0_{M}: S_{i}^{\infty}$. Hence $0_{M}: x \subseteq 0_{M}: S_{i}^{\infty}$. So $x$ is an $S_{i}$-filterregular element with respect to $M$ if and only if

$$
0_{M}: x \subseteq 0_{M}: S_{i}^{\infty} .
$$

REMARK 2.4. From Proposition 2.3, it follows that there is not any strong-filterregular sequence in $\bigcup_{i=1}^{d} S_{i}$ if and only if $S_{+} \subseteq \sqrt{\mathrm{AnnM}}$. In this case, $M_{\left(n_{1}, \ldots, n_{d}\right)}=0$ for $n_{1}+\cdots+n_{d} \gg 0$. Consequently, there is not any strong-filter-regular sequence in $\bigcup_{i=1}^{d} S_{i}$ if and only if $M_{\left(n_{1}, \ldots, n_{d}\right)}=0$ for all $n_{1}+\cdots+n_{d} \gg 0$. 
In the case that $A$ is artinian, $\ell_{A}\left[M_{\left(0, \ldots, 0, n_{i}, 0, \ldots, 0\right)}\right]$ is a polynomial for all $n_{i} \gg 0$. Denote by $P_{i}\left(n_{i}\right)$ the polynomial of $\ell_{A}\left[M_{\left(0, \ldots, 0, n_{i}, 0, \ldots, 0\right)}\right]$.

Now, if we assign the degree -1 to the zero polynomial then we have the following proposition.

PROposition 2.5. Let $A$ be an artinian ring. Then for each $1 \leq i \leq d$, the length of every maximal strong-filter-regular sequence in $S_{i}$ with respect to $M$ is equal to $\operatorname{deg} P_{i}\left(n_{i}\right)+1$.

Proof. Note that if $x \in S_{i}$ is an $S_{i}$-filter-regular element with respect to $M$, then

$$
\ell_{A}\left[(M / x M)_{\left(0, \ldots, 0, n_{i}, 0, \ldots, 0\right)}\right]=\ell_{A}\left[M_{\left(0, \ldots, 0, n_{i}, 0, \ldots, 0\right)}\right]-\ell_{A}\left[M_{\left(0, \ldots, 0, n_{i}-1,0, \ldots, 0\right)}\right]
$$

for all $n_{i} \gg 0$ by [22, Remark 2.6]. On the other hand, it easily seen that $P_{i}\left(n_{i}\right)=0$ if and only if $S_{i} \subseteq \sqrt{\mathrm{Ann} M}$. Hence using the same argument as in the proof of [22, Proposition 3.3(iii)], we get the proof of this proposition.

REMARK 2.6. Proposition 2.5 shows that if $A$ is an artinian ring, then the set of lengths of maximal strong-filter-regular sequences with respect to $M$ is bounded above by $\sum_{i=1}^{d} \operatorname{deg} P_{i}\left(n_{i}\right)+d$.

Now, we study the relationship between filter-regular sequences and weak-(FC)sequences of ideals. This is a kind of variant of superficial sequences.

The author of [15] in 2000 characterized mixed multiplicities as the Hilbert-Samuel multiplicity by (FC)-sequences (see e.g. [2, 3, 7, 16, 17, 18, 19, 20, 24]).

Definition 2.7 ([15]). Let $N$ be a finitely generated $A$-module. Let $I_{1}, \ldots, I_{d}$ be ideals such that $I=I_{1} \ldots I_{d}$ is not contained in $\sqrt{\operatorname{Ann} N}$. An element $x \in A$ is called a weak-(FC)- element of $N$ with respect to $\left(I_{1}, \ldots, I_{d}\right)$ if there exists $i \in\{1, \ldots, d\}$ such that $x \in I_{i}$ and the following conditions are satisfied:

(i) $x$ is an $I$-filter-regular element with respect to $N$, i.e., $0_{N}: x \subseteq 0_{N}: I^{\infty}$.

(ii) $x N \cap I_{1}{ }^{n_{1}} \ldots I_{i}^{n_{i}+1} \ldots I_{d}^{n_{d}} N=x I_{1}{ }^{n_{1}} \ldots I_{i}^{n_{i}} \ldots I_{d}^{n_{d}} N$ for all $n_{1}, \ldots, n_{d} \gg 0$.

Let $x_{1}, \ldots, x_{t}$ be a sequence in $A$. For any $0 \leq i<t$, set $N_{i}=\frac{N}{\left(x_{1}, \ldots, x_{i}\right) N}$. Then $x_{1}, \ldots, x_{t}$ is called a weak-(FC)-sequence of $N$ with respect to $\left(I_{1}, \ldots, I_{d}\right)$ if $x_{i+1}$ is a weak-(FC)-element of $N_{i}$ with respect to $\left(I_{1}, \ldots, I_{d}\right)$ for all $i=0, \ldots, t-1$.

A weak-(FC)-sequence $x_{1}, \ldots, x_{t}$ of $N$ with respect to $\left(I_{1}, \ldots, I_{d}\right)$ is called a maximal weak$(F C)$-sequence if $I \subseteq \sqrt{\operatorname{Ann} N_{t}}$.

Set

$$
\mathcal{S}=F_{I_{1}}\left(I_{1}, \ldots, I_{d} ; A\right)=\bigoplus_{n_{1}, \ldots, n_{d} \geq 0} \frac{I_{1}^{n_{1}} \ldots I_{d}^{n_{d}}}{I_{1}^{n_{1}+1} I_{2}^{n_{2}} \ldots I_{d}^{n_{d}}}
$$


and

$$
\mathcal{M}=F_{I_{1}}\left(I_{1}, \ldots, I_{d} ; N\right)=\bigoplus_{n_{1}, \ldots, n_{d} \geq 0} \frac{I_{1}^{n_{1}} \cdots I_{d}^{n_{d}} N}{I_{1}^{n_{1}+1} I_{2}^{n_{2}} \cdots I_{d}^{n_{d}} N}
$$

Then $\mathcal{S}$ is a finitely generated standard multi-graded algebra over $A / I_{1}$ and $\mathcal{M}$ is a finitely generated multi-graded $\mathcal{S}$-module.

Assume that $x \in I_{i}$ is a weak-(FC)-element of $N$ with respect to $\left(I_{1}, \ldots, I_{d}\right)$ and $x^{*}$ is the image of $x$ in $\mathcal{S}_{i}=\left(F_{I_{1}}\left(I_{1}, \ldots, I_{d} ; A\right)\right)_{i}$. Then by [25, Proposition 4.5(i)], $x^{*}$ is an $\mathcal{S}_{++}=$ $F_{I_{1}}\left(I_{1}, \ldots, I_{d} ; A\right)_{++}$-filter-regular element of $\mathcal{M}$. Note that $I=I_{1} \ldots I_{d} \subseteq \sqrt{\operatorname{Ann}_{A} N}$ if and only if

$$
\mathcal{S}_{(1,1, \ldots, 1)} \subseteq \sqrt{\mathrm{Ann}_{\mathcal{S}} \mathcal{M}}
$$

We immediately get the following result.

PROPOSITION 2.8. Let $x_{1}, \ldots, x_{t}$ be a sequence in $\bigcup_{i=1}^{d} I_{i}$ and $x_{1}^{*}, \ldots, x_{t}^{*}$ the images of $x_{1}, \ldots, x_{t}$ in $\bigcup_{i=1}^{d} \mathcal{S}_{i}$, respectively. Then if $x_{1}, \ldots, x_{t}$ is a weak- $(F C)$-sequence of $N$ with respect to $\left(I_{1}, \ldots, I_{d}\right), x_{1}^{*}, \ldots, x_{t}^{*}$ is an $\mathcal{S}_{++}$-filter-regular sequence with respect to $\mathcal{M}$. In this case, $x_{1}, \ldots, x_{t}$ is a maximal weak-(FC)-sequence of $N$ with respect to $\left(I_{1}, \ldots, I_{d}\right)$ if and only if $x_{1}^{*}, \ldots, x_{t}^{*}$ is a maximal $\mathcal{S}_{++}$-filter-regular sequence with respect to $\mathcal{M}$.

The notion of superficial elements goes back to P. Samuel [26]. The classical theory of superficial elements is an important tool in local algebra and has been continually developed (see e.g. $[4,6,11,13]$ ).

Definition 2.9. Let $N$ be a finitely generated $A$-module. Let $I_{1}, \ldots, I_{d}$ be ideals such that $I=I_{1} \ldots I_{d}$ is not contained in $\sqrt{\operatorname{Ann}_{A} N}$. An element $x$ is called an $I_{i}$-superficial element of $N$ with respect to $\left(I_{1}, \ldots, I_{d}\right)$ if $x \in I_{i}$ and there exists a non-negative integer $c$ such that

$$
\left(I_{1}^{n_{1}} \ldots I_{i}^{n_{i}+1} \ldots I_{d}^{n_{d}} N: x\right) \bigcap I_{1}^{n_{1}} \ldots I_{i}^{c} \ldots I_{d}^{n_{d}} N=I_{1}^{n_{1}} \ldots I_{d}^{n_{d}} N
$$

for all $n_{i} \geq c$ and for all non-negative integers $n_{1}, \ldots, n_{i-1}, n_{i+1}, \ldots, n_{d}$.

Let $x_{1}, \ldots, x_{t}$ be a sequence in $A$. For any $0 \leq i<t$, set $N_{i}=\frac{N}{\left(x_{1}, \ldots, x_{i}\right) N}$. Then $x_{1}, \ldots, x_{t}$ is called a superficial sequence of $N$ with respect to $\left(I_{1}, \ldots, I_{d}\right)$ if $x_{i+1}$ is a superficial element of $N_{i}$ with respect to $\left(I_{1}, \ldots, I_{d}\right)$ for all $i=0, \ldots, t-1$. A superficial sequence $x_{1}, \ldots, x_{t}$ of $N$ with respect to $\left(I_{1}, \ldots, I_{d}\right)$ is called a maximal superficial sequence if $I \subseteq \sqrt{\operatorname{Ann}_{A} N_{t}}$ (see [11]).

Now we need to prove that if $I_{1}, \ldots, I_{d}$ are $\mathfrak{m}$-primary and $x$ is an $I_{i}$-superficial element of $N$ with respect to $\left(I_{1}, \ldots, I_{d}\right)$, then $x$ is a weak- $(F C)$-element of $N$ with respect to $\left(I_{1}, \ldots, I_{d}\right)$. Indeed, by [4, Lemma 17.2.4],

$$
I_{1}^{n_{1}} \ldots I_{d}^{n_{d}} N: x=\left(0_{N}: x\right)+I_{1}^{n_{1}} \ldots I_{i}^{n_{i}-1} \ldots I_{d}^{n_{d}} N
$$


for all $n_{1}, \ldots, n_{s} \gg 0$. Multiplying the last equation by $x$ yields

$$
I_{1}^{n_{1}} \cdots I_{d}^{n_{d}} N \bigcap x N=x I_{1}^{n_{1}} \cdots I_{i}^{n_{i}-1} \cdots I_{d}^{n_{d}} N
$$

for $n_{1}, \ldots, n_{d} \gg 0$. Hence $x$ satisfies the condition (ii) of a weak-( $\left.F C\right)$ - element of $N$ with respect to $\left(I_{1}, \ldots, I_{d}\right)$. By [4, Lemma 17.2.4], we have

$$
\left(0_{N}: x\right) \bigcap I_{1}^{n_{1}} \cdots I_{d}^{n_{d}} N=0
$$

for $n_{1}, \ldots, n_{d} \gg 0$. Hence $0_{N}: x \subseteq 0_{N}: I^{\infty}$. So $x$ is an $I$-filter-regular element with respect to $N$, i.e., $x$ satisfies the condition (i) of a weak- $(F C)$ - element of $N$ with respect to $\left(I_{1}, \ldots, I_{d}\right)$. Thus $x$ is a weak- $(F C)$ - element of $N$ with respect to $\left(I_{1}, \ldots, I_{d}\right)$. Note that a superficial sequence $x_{1}, \ldots, x_{m}$ of $N$ with respect to $\left(I_{1}, \ldots, I_{d}\right)$ is a maximal superficial sequence if and only if $I \subseteq \sqrt{\operatorname{Ann} N_{m}}$. Therefore $x_{1}, \ldots, x_{m}$ is a maximal superficial sequence of $N$ with respect to $\left(I_{1}, \ldots, I_{d}\right)$ if and only if $x_{1}, \ldots, x_{m}$ is also a maximal weak- $(F C)$ sequence of $N$ with respect to $\left(I_{1}, \ldots, I_{d}\right)$.

This fact yields the following.

Proposition 2.10. Let $I_{1}, \ldots, I_{d}$ be $\mathfrak{m}$-primary ideals. Assume that $x_{1}, \ldots, x_{t}$ is a superficial sequence of $N$ with respect to $\left(I_{1}, \ldots, I_{d}\right)$. Then $x_{1}, \ldots, x_{t}$ is a weak- $(F C)$ sequence of $N$ with respect to $\left(I_{1}, \ldots, I_{d}\right)$. In this case, $x_{1}, \ldots, x_{t}$ is a maximal superficial sequence of $N$ with respect to $\left(I_{1}, \ldots, I_{d}\right)$ if and only if $x_{1}, \ldots, x_{t}$ is a maximal weak- $(F C)$ sequence of $N$ with respect to $\left(I_{1}, \ldots, I_{d}\right)$.

Then as an immediate consequence of Proposition 2.8 and Proposition 2.10, we have the following result.

Proposition 2.11. Let $I_{1}, \ldots, I_{d}$ be $\mathfrak{m}$-primary ideals. Assume that $x_{1}, \ldots, x_{t}$ is a sequence in $\bigcup_{i=1}^{d} I_{i}$ and $x_{1}^{*}, \ldots, x_{t}^{*}$ the images of $x_{1}, \ldots, x_{t}$ in $\bigcup_{i=1}^{d} \mathcal{S}_{i}$, respectively. Then if $x_{1}, \ldots, x_{t}$ is a superficial sequence of $N$ with respect to $\left(I_{1}, \ldots, I_{d}\right), x_{1}^{*}, \ldots, x_{t}^{*}$ is an $\mathcal{S}_{++}$filter-regular sequence with respect to $\mathcal{M}$. In this case, $x_{1}, \ldots, x_{t}$ is a maximal superficial sequence of $N$ with respect to $\left(I_{1}, \ldots, I_{d}\right)$ if and only if $x_{1}^{*}, \ldots, x_{t}^{*}$ is a maximal $\mathcal{S}_{++}$-filterregular sequence with respect to $\mathcal{M}$.

REMARK 2.12. Let $I_{1}, \ldots, I_{d}$ be $\mathfrak{m}$-primary ideals. Assume that $x$ is an $I_{i}$-superficial element of $N$ with respect to $\left(I_{1}, \ldots, I_{d}\right)$ and $x^{*}$ the image of $x$ in $\mathcal{S}_{i}$. Then $x^{*}$ is an $\mathcal{S}_{++^{-}}$ filter-regular element with respect to $\mathcal{M}$ by Proposition 2.11. Hence

$$
\left(I_{1}^{n_{1}+1} I_{2}^{n_{2}} \cdots I_{i}^{n_{i}+1} \cdots I_{d}^{n_{d}} N: x\right) \bigcap I_{1}^{n_{1}} \cdots I_{d}^{n_{d}} N=I_{1}^{n_{1}+1} I_{2}^{n_{2}} \cdots I_{d}^{n_{d}} N
$$

for all $n_{1}, \ldots, n_{d} \gg 0$. And by [22, Proposition 2.5], this is equivalent to

$$
x^{*} \in \mathcal{S}_{i} \backslash \bigcup_{P \in \operatorname{Ass}_{\mathcal{S}} \mathcal{M} /\left(0_{\mathcal{M}}: \mathcal{S}_{++}^{\infty}\right)} P .
$$




\section{Filter-Regular Sequences and Joint Reductions}

In this section, we explore the relationship between maximal filter-regular sequences and reductions.

The concept of joint reductions of $\mathfrak{m}$-primary ideals was given by Rees in 1984 [10]. And he proved that mixed multiplicities of $\mathfrak{m}$-primary ideals are multiplicities of ideals generated by joint reductions. This concept was extended to the set of arbitrary ideals by [7, 9, 17, 19]. In the case of graded modules, we have the following.

Definition 3.1. Let $S=\bigoplus_{n_{1}, \ldots, n_{d} \geq 0} S_{\left(n_{1}, \ldots, n_{d}\right)}$ be a finitely generated standard $\mathbf{N}^{d}$ graded algebra over a noetherian local ring $A$ and let $M=\bigoplus_{n_{1}, \ldots, n_{d} \geq 0} M_{\left(n_{1}, \ldots, n_{d}\right)}$ be a finitely generated $\mathbf{N}^{d}$-graded $S$-module. Let $\mathfrak{R}$ be a subset of $\bigcup_{i=1}^{d} S_{i}$. Set $\mathfrak{R}_{i}=\mathfrak{R} \bigcap S_{i}$ for all $i=1, \ldots, d$ and $(\emptyset)=0_{S}$. Then $\Re$ is called a joint reduction of $\left(S_{1}, \ldots, S_{d}\right)$ with respect to $M$ if

$M_{\left(n_{1}+1, \ldots, n_{d}+1\right)}=\sum_{i=1}^{d}\left(\Re_{i}\right) M_{\left(n_{1}+1, \ldots, n_{i}, \ldots, n_{d}+1\right)}$ for all large integers $n_{1}, n_{2}, \ldots, n_{d}$.

Recall that an ideal $\mathfrak{J}$ is called a reduction of an ideal $\mathcal{I}$ with respect to A-module $N$ if $\mathfrak{J} \subseteq \mathcal{I}$ and $\mathcal{I}^{n+1} N=\mathfrak{J} \mathcal{I}^{n} N$ for all large $n$ by [8].

Then the relationship between maximal filter-regular sequences and reductions is stated by the following theorem.

THEOREM 3.2. Let $\mathfrak{R}$ be a subset of $\bigcup_{i=1}^{d} S_{i}$. Then the following statements hold.

(i) If $\Re$ is a maximal $S_{++}$-filter-regular sequence with respect to $M$, then $\Re$ is a joint reduction of $\left(S_{1}, \ldots, S_{d}\right)$ with respect to $M$.

(ii) If $\mathfrak{R}$ is a maximal strong-filter-regular sequence with respect to $M$, then $(\mathfrak{R})$ is a reduction of $S_{+}$with respect to $M$.

PRoOF. Set $\mathfrak{R}_{i}=\mathfrak{R} \bigcap S_{i}$ for all $i=1, \ldots, d$. Assume that $\mathfrak{R}$ is a maximal $S_{++^{-}}$ filter-regular sequence with respect to $M$. Then we have

$$
S_{(1,1, \ldots, 1)} \subseteq \sqrt{\operatorname{Ann}[M /(\Re) M]} .
$$

Hence by Remark 2.2(ii), $[M /(\Re) M]_{\left(n_{1}, \ldots, n_{d}\right)}=0$ for all large $n_{1}, \ldots, n_{d}$. From this it follows that

$$
M_{\left(n_{1}+1, \ldots, n_{d}+1\right)}=\sum_{i=1}^{d}\left(\Re_{i}\right) M_{\left(n_{1}+1, \ldots, n_{i}, \ldots, n_{d}+1\right)}
$$

for all large integers $n_{1}, n_{2}, \ldots, n_{d}$. Thus, $\mathfrak{R}$ is a joint reduction of $\left(S_{1}, \ldots, S_{d}\right)$ with respect to $M$. We have (i). Now, if $\Re$ is a maximal strong-filter-regular sequence with respect to $M$, then it follows that there is not any strong-filter-regular sequence in $\bigcup_{i=1}^{d} S_{i}$ with respect to 
$M /(\mathfrak{R}) M$. Hence by Remark $2.4,[M /(\mathfrak{R}) M]_{\left(n_{1}, \ldots, n_{d}\right)}=0$ for all $n_{1}+\cdots+n_{d} \gg 0$. This is equivalent to

$$
\sum_{i=1}^{d}\left(\Re_{i}\right) M_{\left(n_{1}, \ldots, n_{i}-1, \ldots, n_{d}\right)}=M_{\left(n_{1}, \ldots, n_{d}\right)}
$$

for all $n_{1}+\cdots+n_{d} \gg 0$. Here we assign that $M_{\left(n_{1}, \ldots,-1, \ldots, n_{d}\right)}=0$. Direct computation shows that

$$
S_{+}^{n} M=\sum_{n_{1}+\cdots+n_{d} \geq n} M_{\left(n_{1}, \ldots, n_{d}\right)}
$$

and

$$
\left[(\mathfrak{R}) \sum_{n_{1}+\cdots+n_{d} \geq n} M_{\left(n_{1}, \ldots, n_{d}\right)}\right]_{\left(m_{1}, \ldots, m_{d}\right)}=\sum_{i=1}^{d}\left(\Re_{i}\right) M_{\left(m_{1}, \ldots, m_{i}-1, \ldots, m_{d}\right)}
$$

for all $m_{1}+\cdots+m_{d} \geq n+1$. Hence we have

$$
\begin{aligned}
(\mathfrak{R}) S_{+}^{n} M & =(\mathfrak{R}) \sum_{n_{1}+\cdots+n_{d} \geq n} M_{\left(n_{1}, \ldots, n_{d}\right)} \\
& =\sum_{m_{1}+\cdots+m_{d} \geq n+1}\left[\sum_{i=1}^{d}\left(\Re_{i}\right) M_{\left(m_{1}, \ldots, m_{i}-1, \ldots, m_{d}\right)}\right] \\
& =\sum_{m_{1}+\cdots+m_{d} \geq n+1} M_{\left(m_{1}, \ldots, m_{d}\right)}=S_{+}^{n+1} M
\end{aligned}
$$

for all $n \gg 0$. Thus, $(\mathfrak{R})$ is a reduction of $S_{+}$with respect to $M$. So we get (ii).

By the proof of Theorem 3.2(ii), we immediately obtain the following corollary.

COROLlary 3.3. ( $\mathfrak{R})$ is a reduction of $S_{+}$with respect to $M$ if and only if

$$
\sum_{i=1}^{d}\left(\Re_{i}\right) M_{\left(n_{1}, \ldots, n_{i}-1, \ldots, n_{d}\right)}=M_{\left(n_{1}, \ldots, n_{d}\right)} \text { for all } n_{1}+\cdots+n_{d} \gg 0 .
$$

Now, we consider joint reductions of ideals $I_{1}, \ldots, I_{d}$ with respect to $N$.

Definition 3.4 ([21]). Let $I_{1}, \ldots, I_{d}$ be ideals of $A$ and $N$ an $A$-module. Let $\Re$ be a subset of $\bigcup_{i=1}^{d} I_{i}$. Set $\mathfrak{R} i=\mathfrak{R} \bigcap I_{i}$ for $i=1, \ldots, d$ and $(\emptyset)=0_{A}$. Then $\mathfrak{R}$ is called a joint reduction of $\left(I_{1}, \ldots, I_{d}\right)$ with respect to $N$ if

$$
I_{1}^{n_{1}+1} \cdots I_{d}^{n_{d}+1} N=\sum_{i=1}^{d}\left(\Re_{i}\right) I_{1}^{n_{1}+1} \cdots I_{i}^{n_{i}} \cdots I_{d}^{n_{d}+1} N
$$

for all large integers $n_{1}, n_{2}, \ldots, n_{d}$. 
Recall that $\mathcal{S}=F_{I_{1}}\left(I_{1}, \ldots, I_{d} ; A\right)$ and $\mathcal{M}=F_{I_{1}}\left(I_{1}, \ldots, I_{d} ; N\right)$. Now for each $x \in I_{j}$, denote by $x^{*}$ the image of $x$ in $\mathcal{S}_{j}=\left(F_{I_{1}}\left(I_{1}, \ldots, I_{d} ; A\right)\right)_{j}$. Now, assume that $x_{1}, \ldots, x_{t}$ is a maximal weak-(FC)-sequence of $N$ with respect to $\left(I_{1}, \ldots, I_{d}\right)$ then $x_{1}^{*}, \ldots, x_{t}^{*}$ is a maximal $\mathcal{S}_{++}$-filter-regular sequence with respect to $\mathcal{M}$ by Proposition 2.8. Set

$$
\mathfrak{R}=\left\{x_{1}, \ldots, x_{t}\right\} ; \mathfrak{R}_{i}=\mathfrak{R} \bigcap I_{i}
$$

for all $i=1, \ldots, d$ and

$$
\mathfrak{R}^{*}=\left\{x_{1}^{*}, \ldots, x_{t}^{*}\right\} ; \mathfrak{R}_{i}^{*}=\mathfrak{R}^{*} \bigcap \mathcal{S}_{i}
$$

for all $i=1, \ldots, d$. By Theorem 3.2(i), $\mathfrak{R}^{*}$ is a joint reduction of $\left(\mathcal{S}_{1}, \ldots, \mathcal{S}_{d}\right)$ with respect to $\mathcal{M}$. Hence

$$
\mathcal{M}_{\left(n_{1}+1, \ldots, n_{d}+1\right)}=\sum_{i=1}^{d}\left(\Re_{i}^{*}\right) \mathcal{M}_{\left(n_{1}+1, \ldots, n_{i}, \ldots, n_{d}+1\right)}
$$

for all large integers $n_{1}, n_{2}, \ldots, n_{d}$. From this it follows that

$$
I_{1}^{n_{1}+1} \cdots I_{d}^{n_{d}+1} N=\sum_{i=1}^{d}\left(\Re_{i}\right) I_{1}^{n_{1}+1} \cdots I_{i}^{n_{i}} \cdots I_{d}^{n_{d}+1} N+I_{1}^{n_{1}+2} I_{2}^{n_{2}+1} \cdots I_{d}^{n_{d}+1} N
$$

for all large integers $n_{1}, n_{2}, \ldots, n_{d}$. Therefore,

$$
I_{1}^{n_{1}+1} \cdots I_{d}^{n_{d}+1} N=\sum_{i=1}^{d}\left(\Re_{i}\right) I_{1}^{n_{1}+1} \cdots I_{i}^{n_{i}} \cdots I_{d}^{n_{d}+1} N
$$

for all large integers $n_{1}, n_{2}, \ldots, n_{d}$ by Nakayama's lemma. Thus, $\mathfrak{R}$ is a joint reduction of $\left(I_{1}, \ldots, I_{d}\right)$ with respect to $N$.

Then we get the following result which is a generalization of [19, Theorem 3.4] and [7, Theorem 2.9].

COROLlary 3.5. If $x_{1}, \ldots, x_{t}$ is a maximal weak-(FC)-sequence of $N$ with respect to $\left(I_{1}, \ldots, I_{d}\right)$ then $\left\{x_{1}, \ldots, x_{t}\right\}$ is a joint reduction of $\left(I_{1}, \ldots, I_{d}\right)$ with respect to $N$.

In the case that $I_{1}, \ldots, I_{d}$ are $\mathfrak{m}$-primary ideals then from Proposition 2.10 and Corollary 3.5 , we immediately get the following result.

COROLlary 3.6. Let $I_{1}, \ldots, I_{d}$ be $\mathfrak{m}$-primary ideals of A. Assume that $x_{1}, \ldots, x_{t}$ is a maximal superficial sequence of $N$ with respect to $\left(I_{1}, \ldots, I_{d}\right)$. Then $\left\{x_{1}, \ldots, x_{t}\right\}$ is a joint reduction of $\left(I_{1}, \ldots, I_{d}\right)$ with respect to $N$.

\section{Filter-Regular Sequences and Parameter Systems}

In this section, we characterize the existence of parts of homogeneous parameter systems for $M$ consisting of elements of total degree 1 via the relationship between strong-filter-regular 
sequences and parts of homogeneous parameter systems.

The main result of this section is the following theorem.

THEOREM 4.1. Let $S=\bigoplus_{n_{1}, \ldots, n_{d} \geq 0} S_{\left(n_{1}, \ldots, n_{d}\right)}$ be a finitely generated standard $\mathbf{N}^{d}$ graded algebra over a noetherian local ring $(A, \mathfrak{m})$ and let $M=\bigoplus_{n_{1}, \ldots, n_{d} \geq 0} M_{\left(n_{1}, \ldots, n_{d}\right)}$ be a finitely generated $\mathbf{N}^{d}$-graded $S$-module. Let $k_{1}, k_{2}, \ldots, k_{d}$ be non-negative integers with $k_{1}+k_{2}+\cdots+k_{d}=s \leq \operatorname{dim} M . \operatorname{Set}(\emptyset)=0_{S}$ and

$$
\mathfrak{S}_{\hat{i}}=\left\{S_{1}, \ldots, S_{i-1}, S_{i+1}, \ldots, S_{d}\right\}
$$

for all $1 \leq i \leq d$. Then the following statements are equivalent.

(i) For any $1 \leq i \leq d, \operatorname{dim}_{S} \frac{M}{\left(\mathfrak{U}, S_{i}\right) M} \leq \operatorname{dim}_{S} \frac{M}{(\mathfrak{U}) M}-k_{i}$ for all $\mathfrak{U} \subset \mathfrak{S}_{\hat{i}}$.

(ii) There is a strong-filter-regular sequence $x_{1}, \ldots, x_{s}$ with respect to $M$ consisting of $k_{1}$ elements of $S_{1}, \ldots, k_{d}$ elements of $S_{d}$ such that if $y_{1}, \ldots, y_{k_{i}} \in S_{i}$ is a subsequence of $x_{1}, \ldots, x_{s}$, then

$$
\operatorname{dim}_{S} \frac{M}{\left(\mathfrak{U}, y_{1}, \ldots, y_{k_{i}}\right) M}=\operatorname{dim}_{S} \frac{M}{(\mathfrak{U}) M}-k_{i}
$$

for all $\mathfrak{U} \subset \mathfrak{S}_{\mathfrak{i}_{i}}$ and $1 \leq i \leq d$. And $x_{1}, \ldots, x_{s}$ is a part of a parameter system for M.

(iii) There is a part of a parameter system $x_{1}, \ldots, x_{s}$ for $M$ consisting of $k_{1}$ elements of $S_{1}, \ldots, k_{d}$ elements of $S_{d}$ such that if $y_{1}, \ldots, y_{k_{i}} \in S_{i}$ is a subsequence of $x_{1}, \ldots, x_{s}$, then

$$
\operatorname{dim}_{S} \frac{M}{\left(\mathfrak{U}, y_{1}, \ldots, y_{k_{i}}\right) M}=\operatorname{dim}_{S} \frac{M}{(\mathfrak{U}) M}-k_{i}
$$

for all $\mathfrak{U} \subset \mathfrak{S}_{\hat{i}}$ and $1 \leq i \leq d$.

PROOF. (i) $\Rightarrow$ (ii): Set $k_{1}+k_{2}+\cdots+k_{d}=s$. The proof is by induction on $s$ that there exists a strong-filter-regular sequence $x_{1}, \ldots, x_{s}$ with respect to $M$ consisting of $k_{1}$ elements of $S_{1}, \ldots, k_{d}$ elements of $S_{d}$ such that if $y_{1}, \ldots, y_{k_{i}} \in S_{i}$ is a subsequence of $x_{1}, \ldots, x_{s}$ then

$$
\operatorname{dim}_{S} \frac{M}{\left(\mathfrak{U}, y_{1}, \ldots, y_{k_{i}}\right) M}=\operatorname{dim}_{S} \frac{M}{(\mathfrak{U}) M}-k_{i}
$$

for all $\mathfrak{U} \subset \mathfrak{S}_{\widehat{i}}$ and $1 \leq i \leq d$. And $x_{1}, \ldots, x_{s}$ is a part of a parameter system for $M$. The result is true for $s=0$. Assume the result for $s-1 \geq 0$. Since $s>0$, there exists $1 \leq v \leq d$ such that $k_{v}>0$. Then we have

$$
\operatorname{dim}_{S} \frac{M}{\left(\mathfrak{U}, S_{v}\right) M} \leq \operatorname{dim}_{S} \frac{M}{(\mathfrak{U}) M}-k_{v}<\operatorname{dim}_{S} \frac{M}{(\mathfrak{U}) M}
$$

for all $\mathfrak{U} \subset \mathfrak{S}_{\widehat{v}}$. Set

$$
U=\operatorname{Ass}_{S} \frac{M}{\left(0_{M}: S_{v}^{\infty}\right)}=\left\{P \in \operatorname{Ass}_{S} M \mid S_{v} \nsubseteq P\right\} .
$$


And for any $\mathfrak{U} \subset \mathfrak{S}_{\widehat{v}}$, set

$$
V_{\mathfrak{U}}=\left\{P \in \operatorname{Ass}_{S} \frac{M}{(\mathfrak{U}) M} \mid \operatorname{dim}_{S} \frac{M}{(\mathfrak{U}) M}=\operatorname{Coht} P\right\} .
$$

Since

$$
\operatorname{dim}_{S} \frac{M}{\left(\mathfrak{U}, S_{v}\right) M}<\operatorname{dim}_{S} \frac{M}{(\mathfrak{U}) M}
$$

$S_{v} \nsubseteq P$ for any $P \in V_{\mathfrak{U}}$ and all $\mathfrak{U} \subset \mathfrak{S}_{\widehat{v}}$. Put $G=\left(\bigcup_{\mathfrak{U} \subset \mathfrak{S}_{\widehat{v}}} V_{\mathfrak{U}}\right) \cup U$. Then $S_{v} \nsubseteq P$ for any $P \in G$. Since $k$ is an infinite field and $G$ is finite, there exists

$$
x \in S_{v} \backslash \bigcup_{P \in G} P .
$$

Because $x \notin \bigcup_{P \in U} P, x$ is an $S_{v}$-filter-regular element with respect to $M$. And since $x \notin$ $\bigcup_{P \in V_{\mathfrak{U}}} P$, it follows that

$$
\operatorname{dim}_{S} \frac{M}{(\mathfrak{U}, x) M}=\operatorname{dim}_{S} \frac{M}{(\mathfrak{U}) M}-1
$$

for all $\mathfrak{U} \subset \mathfrak{S}_{\widehat{v}}$. In particular, $\mathfrak{U}=\emptyset$ then we get

$$
\operatorname{dim}_{S} \frac{M}{x M}=\operatorname{dim}_{S} \frac{M}{(\emptyset, x) M}=\operatorname{dim}_{S} \frac{M}{(\emptyset) M}-1=\operatorname{dim}_{S} M-1 .
$$

Hence $x$ is a parameter element for $M$. Set $\bar{M}=M / x M$ and

$$
h_{1}=k_{1}, \ldots, h_{v-1}=k_{v-1}, h_{v}=k_{v}-1, h_{v+1}=k_{v+1}, \ldots, h_{d}=k_{d} .
$$

Then $h_{1}+\cdots+h_{d}=s-1$. Now, we need to show that

$$
\operatorname{dim}_{S} \frac{\bar{M}}{\left(\mathfrak{U}, S_{i}\right) \bar{M}} \leq \operatorname{dim}_{S} \frac{\bar{M}}{(\mathfrak{U}) \bar{M}}-h_{i}
$$

for all $\mathfrak{U} \subset \mathfrak{S}_{\hat{i}}$ and $1 \leq i \leq d$. Indeed, for $i \neq v$, let $\mathfrak{U} \subset \mathfrak{S}_{\widehat{i}_{i}}$. If $S_{v} \in \mathfrak{U}$ then we have

$$
\operatorname{dim}_{S} \frac{\bar{M}}{\left(\mathfrak{U}, S_{i}\right) \bar{M}}=\operatorname{dim}_{S} \frac{M}{\left(\mathfrak{U}, S_{i}\right) M} \leq \operatorname{dim}_{S} \frac{M}{(\mathfrak{U}) M}-k_{i}=\operatorname{dim}_{S} \frac{\bar{M}}{(\mathfrak{U}) \bar{M}}-h_{i} .
$$

If $S_{v} \notin \mathfrak{U}$ then $\mathfrak{U} \cup\left\{S_{i}\right\} \subset \mathfrak{S}_{\widehat{v}}$. Hence

$$
\begin{aligned}
\operatorname{dim}_{S} \frac{\bar{M}}{\left(\mathfrak{U}, S_{i}\right) \bar{M}} & =\operatorname{dim}_{S} \frac{M}{\left(\mathfrak{U}, S_{i}, x\right) M}=\operatorname{dim}_{S} \frac{M}{\left(\mathfrak{U}, S_{i}\right) M}-1 \\
& \leq \operatorname{dim}_{S} \frac{M}{(\mathfrak{U}) M}-k_{i}-1=\operatorname{dim}_{S} \frac{M}{(\mathfrak{U}, x) M}-k_{i} \\
& =\operatorname{dim}_{S} \frac{\bar{M}}{(\mathfrak{U}) \bar{M}}-h_{i} .
\end{aligned}
$$


For $i=v$,

$$
\begin{aligned}
\operatorname{dim}_{S} \frac{\bar{M}}{\left(\mathfrak{U}, S_{v}\right) \bar{M}} & =\operatorname{dim}_{S} \frac{M}{\left(\mathfrak{U}, S_{v}\right) M} \\
& \leq \operatorname{dim}_{S} \frac{M}{(\mathfrak{U}) M}-k_{v}=\operatorname{dim}_{S} \frac{M}{(\mathfrak{U}, x) M}+1-k_{v} \\
& =\operatorname{dim}_{S} \frac{\bar{M}}{(\mathfrak{U}) \bar{M}}+1-k_{v}=\operatorname{dim}_{S} \frac{\bar{M}}{(\mathfrak{U}) \bar{M}}-h_{v}
\end{aligned}
$$

for all $\mathfrak{U} \subset \mathfrak{S}_{\widehat{v}}$. Consequently $\operatorname{dim}_{S} \frac{\bar{M}}{\left(\mathfrak{U}, S_{i}\right) \bar{M}} \leq \operatorname{dim}_{S} \frac{\bar{M}}{(\mathfrak{U}) \bar{M}}-h_{i}$ for all $\mathfrak{U} \subset \mathfrak{S}_{\hat{i}}$ and $1 \leq i \leq d$.

By induction, there exists a strong-filter-regular sequence $x_{2}, \ldots, x_{s}$ with respect to $\bar{M}$ consisting of $h_{1}$ elements of $S_{1}, \ldots, h_{d}$ elements of $S_{d}$ and if $y_{1}, \ldots, y_{h_{i}} \in S_{i}$ is a subsequence of $x_{2}, \ldots, x_{s}$ then

$$
\operatorname{dim}_{S} \frac{\bar{M}}{\left(\mathfrak{U}, y_{1}, \ldots, y_{h_{i}}\right) \bar{M}}=\operatorname{dim}_{S} \frac{\bar{M}}{(\mathfrak{U}) \bar{M}}-h_{i}
$$

for all $\mathfrak{U} \subset \mathfrak{S}_{\hat{i}}$ and $1 \leq i \leq d$. And $x_{2}, \ldots, x_{s}$ is a part of a parameter system for $\bar{M}$. It is a plain fact that $x=x_{1}, x_{2}, \ldots, x_{s}$ is a strong-filter-regular sequence consisting of $k_{1}=h_{1}$ elements of $S_{1}, \ldots, k_{v-1}=h_{v-1}$ elements of $S_{v-1}, k_{v}=h_{v}+1$ elements of $S_{v}, k_{v+1}=h_{v+1}$ elements of $S_{v+1}, \ldots, k_{d}=h_{d}$ elements of $S_{d}$. Next we need to prove that for any $i$ and $z_{1}, \ldots, z_{k_{i}} \in S_{i}$ is a subsequence of $x_{1}, \ldots, x_{s}$, then

$$
\operatorname{dim}_{S} \frac{M}{\left(\mathfrak{U}, z_{1}, \ldots, z_{k_{i}}\right) M}=\operatorname{dim}_{S} \frac{M}{(\mathfrak{U}) M}-k_{i}
$$

for all $\mathfrak{U} \subset \mathfrak{S}_{\hat{i}}$ and $1 \leq i \leq d$. Indeed, consider the following cases:

The case that $i \neq v$ : Then in this case, $k_{i}=h_{i}$. Let $\mathfrak{U} \subset \mathfrak{S}_{\hat{i}}$.

If $S_{v} \in \mathfrak{U}$ then since $x \in S_{v}$,

$$
\begin{aligned}
\operatorname{dim}_{S} \frac{M}{\left(\mathfrak{U}, z_{1}, \ldots, z_{k_{i}}\right) M} & =\operatorname{dim}_{S} \frac{\bar{M}}{\left(\mathfrak{U}, z_{1}, \ldots, z_{h_{i}}\right) \bar{M}} \\
& =\operatorname{dim}_{S} \frac{\bar{M}}{(\mathfrak{U}) \bar{M}}-h_{i}=\operatorname{dim}_{S} \frac{M}{(\mathfrak{U}) M}-k_{i}
\end{aligned}
$$

If $S_{v} \notin \mathfrak{U}$ then $\mathfrak{U} \subset \mathfrak{S}_{\widehat{v}}$. Hence

$$
\begin{aligned}
\operatorname{dim}_{S} \frac{M}{(\mathfrak{U}) M}-k_{i}-1 & \leq \operatorname{dim}_{S} \frac{M}{\left(\mathfrak{U}, z_{1}, \ldots, z_{k_{i}}\right) M}-1 \\
& \leq \operatorname{dim}_{S} \frac{M}{\left(\mathfrak{U}, x, z_{1}, \ldots, z_{k_{i}}\right) M}=\operatorname{dim}_{S} \frac{\bar{M}}{\left(\mathfrak{U}, z_{1} \ldots, z_{k_{i}}\right) \bar{M}}
\end{aligned}
$$




$$
=\operatorname{dim}_{S} \frac{\bar{M}}{(\mathfrak{U}) \bar{M}}-h_{i}=\operatorname{dim}_{S} \frac{M}{(\mathfrak{U}, x) M}-k_{i}=\operatorname{dim}_{S} \frac{M}{(\mathfrak{U}) M}-k_{i}-1 .
$$

So $\operatorname{dim}_{S} \frac{M}{\left(\mathfrak{U}, z_{1}, \ldots, z_{k_{i}}\right) M}=\operatorname{dim}_{S} \frac{M}{(\mathfrak{U}) M}-k_{i}$.

The case that $i=v$ : In this case, $x=z_{1}$ and $k_{v}=h_{v}+1$. We get

$$
\begin{aligned}
\operatorname{dim}_{S} \frac{M}{\left(\mathfrak{U}, z_{1}, \ldots, z_{k_{v}}\right) M} & =\operatorname{dim}_{S} \frac{\bar{M}}{\left(\mathfrak{U}, z_{2}, \ldots, z_{k_{v}}\right) \bar{M}} \\
& =\operatorname{dim}_{S} \frac{\bar{M}}{(\mathfrak{U}) \bar{M}}-h_{v}=\operatorname{dim}_{S} \frac{\bar{M}}{(\mathfrak{U}) \bar{M}}+1-k_{v} \\
& =\operatorname{dim}_{S} \frac{M}{(\mathfrak{U}, x) M}+1-k_{v}=\operatorname{dim}_{S} \frac{M}{(\mathfrak{U}) M}-k_{v},
\end{aligned}
$$

for all $\mathfrak{U} \subset \mathfrak{S}_{\widehat{v}}$. Therefore

$$
\operatorname{dim}_{S} \frac{M}{\left(\mathfrak{U}, z_{1}, \ldots, z_{k_{i}}\right) M}=\operatorname{dim}_{S} \frac{M}{(\mathfrak{U}) M}-k_{i}
$$

for all $\mathfrak{U} \subset \mathfrak{S}_{\hat{i}}$ and $1 \leq i \leq d$. Now observe that since $x=x_{1}$ is a parameter element for $M$ and $x_{2}, \ldots, x_{s}$ is a part of a parameter system for $M / x M, x_{1}, \ldots, x_{s}$ is a part of a parameter system for $M$. The induction is complete. We get (i) $\Rightarrow$ (ii). (ii) $\Rightarrow$ (iii) is obvious. (iii) $\Rightarrow$ (i): By (iii) we have $\operatorname{dim}_{S} \frac{M}{\left(\mathfrak{U}, y_{1}, \ldots, y_{k_{i}}\right) M}=\operatorname{dim}_{S} \frac{M}{(\mathfrak{U}) M}-k_{i}$ for all $\mathfrak{U} \subset \mathfrak{S}_{\hat{i}}$ and $1 \leq i \leq d$. Hence

$$
\operatorname{dim}_{S} \frac{M}{\left(\mathfrak{U}, S_{i}\right) M} \leq \operatorname{dim}_{S} \frac{M}{\left(\mathfrak{U}, y_{1}, \ldots, y_{k_{i}}\right) M}=\operatorname{dim}_{S} \frac{M}{(\mathfrak{U}) M}-k_{i}
$$

for all $\mathfrak{U} \subset \mathfrak{S}_{\hat{i}}$ and $1 \leq i \leq d$. So (iii) $\Rightarrow$ (i) is proved.

COROLLARY 4.2. Let $S$ be a finitely generated standard $\mathbf{N}^{d}$-graded algebra over a noetherian local ring $(A, \mathfrak{m})$ and let $M$ be a finitely generated $\mathbf{N}^{d}$-graded $S$-module. Set $S_{0}=0_{S}$ and $s=\operatorname{dim}_{S} M-\operatorname{dim}_{A} M_{(0, \ldots, 0)}$ and

$$
k_{i}=\operatorname{dim}_{S} \frac{M}{\left(S_{0}, \ldots, S_{i-1}\right) M}-\operatorname{dim}_{S} \frac{M}{\left(S_{0}, \ldots, S_{i}\right) M}
$$

for any $1 \leq i \leq d$. Assume that

$$
k_{i} \leq \min \left\{\operatorname{dim}_{S} \frac{M}{(\mathfrak{U}) M}-\operatorname{dim}_{S} \frac{M}{\left(\mathfrak{U}, S_{i}\right) M} \mid \emptyset \neq \mathfrak{U} \subset \mathfrak{S}_{\hat{i}}\right\}
$$

for any $1 \leq i \leq d$. Then $k_{1}+\cdots+k_{d}=s$ and the following conditions are equivalent:

(i) $k_{i} \leq \operatorname{dim}_{S} M-\operatorname{dim}_{S} \frac{M}{\left(S_{i}\right) M}$ for all $1 \leq i \leq d$.

(ii) There is a strong-filter-regular sequence with respect to $M$ consisting of $k_{1}$ elements of $S_{1}, \ldots, k_{d}$ elements of $S_{d}$. And this sequence is a part of a parameter system for $M$. 
(iii) There is a part of a homogeneous parameter system for $M$ consisting of $k_{1}$ elements of $S_{1}, \ldots, k_{d}$ elements of $S_{d}$.

PROOF. Since $k_{i}=\operatorname{dim}_{S} \frac{M}{\left(S_{0}, \ldots, S_{i-1}\right) M}-\operatorname{dim}_{S} \frac{M}{\left(S_{0}, \ldots, S_{i}\right) M}$ for any $1 \leq i \leq d, k_{1}+$ $\cdots+k_{d}=\operatorname{dim}_{S} M-\operatorname{dim}_{S} \frac{M}{\left(S_{0}, \ldots, S_{d}\right) M}=\operatorname{dim}_{S} M-\operatorname{dim}_{A} M_{(0, \ldots, 0)}=s$. (i) $\Rightarrow$ (ii): By (i), for any $1 \leq i \leq d$, we have $\operatorname{dim}_{S} \frac{M}{\left(\mathfrak{U}, S_{i}\right) M} \leq \operatorname{dim}_{S} \frac{M}{(\mathfrak{U}) M}-k_{i}$ for all $\mathfrak{U} \subset \mathfrak{S}_{\hat{i}}$. Hence (i) $\Rightarrow$ (ii) is immediate from (i) $\Rightarrow$ (ii) of Theorem 4.1. And (ii) $\Rightarrow$ (iii) is obvious. (iii) $\Rightarrow$ (i): Assume that $x_{1}, \ldots, x_{s} \in \bigcup_{i=1}^{d} S_{i}$ is a part of a parameter system for $M$ consisting of $k_{1}$ elements of $S_{1}, \ldots, k_{d}$ elements of $S_{d}$ and $z_{1}, \ldots, z_{k_{i}} \in S_{i}$ is a subsequence of $x_{1}, \ldots, x_{s}$, then $\operatorname{dim}_{S} \frac{M}{\left(S_{i}\right) M} \leq \operatorname{dim}_{S} \frac{M}{\left(z_{1}, \ldots, z_{i}\right) M}=\operatorname{dim}_{S} M-k_{i}$. Thus, $k_{i} \leq \operatorname{dim}_{S} M-\operatorname{dim}_{S} \frac{M}{\left(S_{i}\right) M}$ for all $1 \leq i \leq d$.

In particular, if $d=1$ then since

$$
\operatorname{dim}_{S} \frac{M}{S_{0} M}-\operatorname{dim}_{S} \frac{M}{\left(S_{0}, S_{1}\right) M}=\operatorname{dim}_{S} M-\operatorname{dim}_{S} \frac{M}{\left(S_{1}\right) M}=\operatorname{dim}_{S} M-\operatorname{dim}_{A} M_{0},
$$

we have the following.

COROLlary 4.3. Let $S=\bigoplus_{n \geq 0} S_{n}$ be a finitely generated standard $\mathbf{N}$-graded algebra over a noetherian local ring $(A, \mathfrak{m})$ and let $M=\bigoplus_{n \geq 0} M_{n}$ be a finitely generated $\mathbf{N}$-graded $S$-module. Set $s=\operatorname{dim}_{S} M-\operatorname{dim}_{A} M_{0}$. Then there is at most s parameter elements for $M$ contained in $S_{1}$.

EXAMPLE 4.4. Let $I$ be an ideal of positive height in a noetherian local ring $(A, \mathfrak{m})$. Denote by $R(I)=\bigoplus_{n \geq 0} I^{n} t^{n}$ the Rees algebra and $G(I)=\bigoplus_{n \geq 0}\left(I^{n} / I^{n+1}\right)$ the associated graded ring of $A$ with respect to $I$. Since $\operatorname{dim} R(I)-\operatorname{dim} A=1$, there is at most one parameter element for $R(I)$ contained in $I t$ by Corollary 4.3. And since $\operatorname{dim} G(I)-\operatorname{dim}(A / I)=$ $\operatorname{dim} A-\operatorname{dim}(A / I)$, there is at most $\operatorname{dim} A-\operatorname{dim}(A / I)$ parameter elements for $G(I)$ contained in $I / I^{2}$ by Corollary 4.3 .

Now, if choose $k_{i}=\min \left\{\operatorname{dim}_{S} \frac{M}{(\mathfrak{U}) M}-\operatorname{dim}_{S} \frac{M}{\left(\mathfrak{U}, S_{i}\right) M} \mid \mathfrak{U} \subset \mathfrak{S}_{\hat{i}}\right\}$ for all $1 \leq i \leq d$, then as an immediate consequence of Theorem 4.1, we have the following consequence.

COROLLARY 4.5. Let $S$ be a finitely generated standard $\mathbf{N}^{d}$-graded algebra over a noetherian local ring $(A, \mathfrak{m})$ and let $M$ be a finitely generated $\mathbf{N}^{d}$-graded $S$-module. Set $k_{i}=\min \left\{\operatorname{dim}_{S} \frac{M}{(\mathfrak{U}) M}-\operatorname{dim}_{S} \frac{M}{\left(\mathfrak{U}, S_{i}\right) M} \mid \mathfrak{U} \subset \mathfrak{S}_{\hat{i}}\right\}$ for any $1 \leq i \leq d$. Then there is a strongfilter-regular sequence with respect to $M$ consisting of $k_{1}$ elements of $S_{1}, \ldots, k_{d}$ elements of $S_{d}$. And this sequence is a part of a parameter system for $M$.

EXAMPLE 4.6. Assume that $\operatorname{dim} A>0 ; J, I_{1}, \ldots, I_{d}(d>1)$ are $\mathfrak{m}$-primary ideals. Denote by $F_{J}\left(I_{1}, \ldots, I_{d} ; A\right)=\bigoplus_{n_{1}, \ldots, n_{d} \geq 0} \frac{I_{1}^{n_{1}} \ldots I_{d}^{n_{d}}}{J I_{1}^{n_{1}} \ldots I_{d}^{n_{d}}}$ the $d$-graded fiber cone of $A$ with 
respect to $J, I_{1}, \ldots, I_{d} . \operatorname{Set} \mathbf{S}=F_{J}\left(I_{1}, \ldots, I_{d} ; A\right) ; \mathbf{S}_{i}=\mathbf{S}_{(\underbrace{0, \ldots, 1}_{i}, \ldots, 0)}=I_{i} / J I_{i}$ and

$$
\mathfrak{S}_{\hat{i}}^{*}=\left\{\mathbf{S}_{1}, \ldots, \mathbf{S}_{i-1}, \mathbf{S}_{i+1}, \ldots, \mathbf{S}_{d}\right\} \text { for all } 1 \leq i \leq d .
$$

Then $\operatorname{dim} \mathbf{S}=\operatorname{dim} A+d-1$ (see [23]). Since $\frac{\mathbf{S}}{\left(\mathbf{S}_{i}\right)} \cong F_{J}\left(I_{1}, \ldots, I_{i-1}, I_{i+1}, \ldots, I_{d} ; A\right)$ and $d>1, \operatorname{dim} \frac{\mathbf{S}}{\left(\mathbf{S}_{i}\right)}=\operatorname{dim} A+d-2 . \operatorname{Sod} \operatorname{dim} \mathbf{S}-\operatorname{dim} \frac{\mathbf{S}}{\left(\mathbf{S}_{i}\right)}=1$ for all $1 \leq i \leq d$. Now, assume that there exists a parameter system for $\mathbf{S}$ consisting of $(\operatorname{dim} A+d-1)$ elements of $\bigcup_{i=1}^{d} \mathbf{S}_{i}$. If $\operatorname{dim} A>1$ then there exists $i$ such that $\mathbf{S}_{i}$ contains two parameter elements $x, y$. In this case,

$$
1=\operatorname{dim} \mathbf{S}-\operatorname{dim} \frac{\mathbf{S}}{\left(\mathbf{S}_{i}\right)} \geq \operatorname{dim} \mathbf{S}-\operatorname{dim} \frac{\mathbf{S}}{(x, y)}=2 .
$$

This contradiction shows that if $\operatorname{dim} A>1$, then there does not exist a homogeneous parameter ideal for $\mathbf{S}$ generated by elements of total degree 1. However, since

$$
1=\min \left\{\operatorname{dim}_{\mathbf{S}} \frac{\mathbf{S}}{(\mathfrak{U})}-\operatorname{dim}_{\mathbf{S}} \frac{\mathbf{S}}{\left(\mathfrak{U}, \mathbf{S}_{i}\right)} \mid \mathfrak{U} \subset \mathfrak{S}_{i}^{*}\right\}
$$

for any $1 \leq i \leq d$, there exists a part of a parameter system for $\mathbf{S}$ consisting of 1 element of $\mathbf{S}_{1}, \ldots, 1$ element of $\mathbf{S}_{d}$ by Corollary 4.5(ii). Thus, in this case, there is a homogeneous parameter ideal for $\mathbf{S}$ generated by homogeneous elements of total degree 1 if and only if $\operatorname{dim} A=1$.

COROLlary 4.7. Let $(A, \mathfrak{m})$ be an artinian local ring. Let $S$ be a finitely generated standard $\mathbf{N}^{d}$-graded algebra over $A$ and let $M$ be a finitely generated $\mathbf{N}^{d}$-graded $S$-module. Set $S_{0}=0_{S}$ and

$$
k_{i}=\operatorname{dim}_{S} \frac{M}{\left(S_{0}, \ldots, S_{i-1}\right) M}-\operatorname{dim}_{S} \frac{M}{\left(S_{0}, \ldots, S_{i}\right) M}
$$

for any $1 \leq i \leq d$. Assume that

$$
k_{i} \leq \min \left\{\operatorname{dim}_{S} \frac{M}{(\mathfrak{U}) M}-\operatorname{dim}_{S} \frac{M}{\left(\mathfrak{U}, S_{i}\right) M} \mid \emptyset \neq \mathfrak{U} \subset \mathfrak{S}_{\hat{i}}\right\}
$$

for any $1 \leq i \leq d$. Then there is a parameter system for $M$ consisting of $k_{1}$ elements of $S_{1}, \ldots, k_{d}$ elements of $S_{d}$ if and only if $k_{i} \leq \operatorname{dim}_{S} M-\operatorname{dim}_{S} \frac{M}{\left(S_{i}\right) M}$ for all $1 \leq i \leq d$.

Proof. Since $A$ is an artinian ring,

$$
k_{1}+\cdots+k_{d}=\sum_{i=1}^{d}\left[\operatorname{dim}_{S} \frac{M}{\left(S_{0}, \ldots, S_{i-1}\right) M}-\operatorname{dim}_{S} \frac{M}{\left(S_{0}, \ldots, S_{i}\right) M}\right]=\operatorname{dim}_{S} M .
$$

Hence the proof follows from Corollary 4.2. 
COROLlary 4.8. Let $(A, \mathfrak{m})$ be an artinian local ring. Let $S$ be a finitely generated standard $\mathbf{N}^{d}$-graded algebra over $A$ and let $M$ be a finitely generated $\mathbf{N}^{d}$-graded $S$-module. Set $S_{0}=0_{S}$ and

$$
k_{i}=\operatorname{dim}_{S} \frac{M}{\left(S_{0}, \ldots, S_{i-1}\right) M}-\operatorname{dim}_{S} \frac{M}{\left(S_{0}, \ldots, S_{i}\right) M}
$$

for all $1 \leq i \leq d$. Assume that

$$
k_{i} \leq \min \left\{\operatorname{dim}_{S} \frac{M}{(\mathfrak{U}) M}-\operatorname{dim}_{S} \frac{M}{\left(\mathfrak{U}, S_{i}\right) M} \mid \emptyset \neq \mathfrak{U} \subset \mathfrak{S}_{\hat{i}}\right\}
$$

for any $1 \leq i \leq d$. Then there is a maximal strong-filter-regular sequence $\mathfrak{R}$ with respect to $M$ consisting of $k_{1}$ elements of $S_{1}, \ldots, k_{d}$ elements of $S_{d}$ if and only if

$$
k_{i} \leq \operatorname{dim}_{S} M-\operatorname{dim}_{S} \frac{M}{\left(S_{i}\right) M}
$$

for all $1 \leq i \leq d$. And in this case, $\mathfrak{R}$ is a parameter system for $M$.

PROOF. The "if" part: By Corollary 4.2, there is a strong-filter-regular sequence with respect to $M$ consisting of $k_{1}$ elements of $S_{1}, \ldots, k_{d}$ elements of $S_{d}$. And this sequence is a part of a parameter system for $M$. Since $k_{1}+\cdots+k_{d}=\operatorname{dim}_{S} M$, this sequence is a parameter system for $M$. Hence it is a maximal strong-filter-regular sequence. The "only if" part: Assume that $\mathfrak{R}$ is a maximal strong-filter-regular sequence with respect to $M$ consisting of $k_{1}$ elements of $S_{1}, \ldots, k_{d}$ elements of $S_{d}$. By Theorem 3.2(ii), (R) is a reduction of $S_{+}$ with respect to $M$. Set $\operatorname{dim}_{S} M=s$. Since $|\mathfrak{R}|=s, \mathfrak{R}$ is a parameter system for $M$. Thus, $k_{i} \leq \operatorname{dim}_{S} M-\operatorname{dim}_{S} \frac{M}{\left(S_{i}\right) M}$ for all $1 \leq i \leq d$ by Corollary 4.7 .

\section{References}

[1] M. Brodmann and R. Y. Sharp, Local Cohomology: An algebraic Introduction with Geometric Applications, Cambridge studies in advanced mathematics, No 60, Cambridge University Press, 1998.

[2] R. CAllejas-Bedregal and V. H. Jorge PÉReZ, (FC)-Sequences, mixed multiplicities and reductions of modules, arXiv:1109.5058 (2011).

[ 3 ] L. V. Dinh and D. Q. VIET, On two results of mixed multiplicities, Int. J. Algebra 4(1) (2010), 19-23.

[ 4 ] C. Huneke and I. SWAnson, Integral Closure of Ideals, Rings, and Modules, London Mathematical Lecture Note Series 336, Cambridge University Press, 2006.

[ 5 ] E. HYRY, The diagonal subring and the Cohen-Macaulay property of a multigraded ring, Trans. Amer. Math. Soc. 351 (1999), 2213-2232.

[6] D. KIRBY, A note on superficial elements of an ideal in a local ring, Quart. J. Math. Oxford (2) 14 (1963), 21-28.

[ 7 ] N. T. Manh and D. Q. ViET, Mixed multiplicities of modules over noetherian local rings, Tokyo J. Math. 29 (2006), 325-345.

[ 8 ] D. G. NorthcotT and D. Rees, Reduction of ideals in local rings, Proc. Cambridge Phil. Soc. 50 (1954), 145-158. 
[9] L. O'CARroll, On two theorems concerning reductions in local rings, J. Math. Kyoto Univ. 27-1 (1987), 61-67.

[10] D. REES, Generalizations of reductions and mixed multiplicities, J. London Math. Soc. 29 (1984), $397-414$.

[11] M. E. Rossi and G. VALlA, Hilbert function of filtered modules, arXiv: 0710.2346 (2008).

[12] J. Stuckrad and W. Vogel, Buchsbaum Rings and Applications, VEB Deutscher Verlag der Wisssenschaften, Berlin, 1986.

[13] B. TeISIER, Cycles èvanescents, sections planes, et conditions de Whitney, Singularities à Cargése Astérisque, 7-8 (1973), 285-362.

[14] N. V. TRung, Reduction exponents and degree bound for the defining equation of graded rings, Proc. Amer. Mat. Soc. 101 (1987), 229-234.

[15] D. Q. Viet, Mixed multiplicities of arbitrary ideals in local rings, Comm. Algebra 28 (2000), 3803-3821.

[16] D. Q. VIET, On some properties of $(F C)$-sequences of ideals in local rings, Proc. Amer. Math. Soc. 131 (2003), 45-53.

[17] D. Q. VIET, Sequences determining mixed multiplicities and reductions of ideals, Comm. Algebra 31 (2003), 5047-5069.

[18] D. Q. VIET, On the mixed multiplicity and the multiplicity of blow-up rings of equimultiple ideals, J. Pure and Appl. Algebra 183 (2003), 313-327.

[19] D. Q. VIET, Reductions and mixed multiplicities of ideals, Comm. Algebra 32 (2004), 4159-4178.

[20] D. Q. VIET, The multiplicity and the Cohen-Macaulayness of extended Rees algebras of equimultiple ideals, J. Pure and Appl. Algebra 205 (2006), 498-509.

[21] D. Q. Viet, L. V. Dinh and T. T. H. Thanh, A note on joint reductions and mixed multiplicities, Proc. Amer. Math. Soc. 142 (2014), 1861-1873.

[22] D. Q. ViET and N. T. MANH, Mixed multiplicities of multigraded modules, Forum Math. 25 (2013), 337-361.

[23] D. Q. ViET and N. T. MANH, On the multiplicity of multigraded modules over Artinian local rings, Tokyo J. Math. 33 (2010), 341-360.

[24] D. Q. VIET and T. T. H. Thanh, On $(F C)$-sequences and mixed multiplicities of multi-graded algebras, Tokyo J. Math. 34 (2011), 185-202.

[25] D. Q. Viet and T. T. H. Thanh, On some multiplicity and mixed multiplicity formulas, Forum Math. 26 (2014), 413-442.

[26] O. ZARISKI and P. S AMUel, Commutative Algebra, Vol II, Van Nostrand, New York, 1960.

\author{
Present Addresses: \\ DUONG QUOC VIET \\ DEPARTMENT OF MATHEMATICS, \\ HANOI NATIONAL UNIVERSITY OF EDUCATION, \\ 136 Xuan Thuy Street, Hanoi, Vietnam. \\ e-mail:vietdq@hnue.edu.vn \\ TRUONG THI HONG THANH \\ DEPARTMENT OF MATHEMATICS, \\ HANOI NATIONAL UNIVERSITY OF EDUCATION, \\ 136 XuAn Thuy STREet, HaNoi, Vietnam. \\ e-mail: thanhtth@hnue.edu.vn
}

